\title{
Lower bounds of heights of points on hypersurfaces
}

\author{
by \\ Frits Beukers (Utrecht) and Don ZaGier (Bonn) \\ To J. W. S. Cassels with respect and admiration
}

1. Introduction. Let us first recall Lehmer's conjecture [Le] on lower bounds for the height of an algebraic number which was stated in 1933. Let $K$ be an algebraic number field of degree $D$ over $\mathbb{Q}$. For any valuation $v$ we define $D_{v}=\left[K_{v}: \mathbb{Q}_{v}\right]$, where $K_{v}, \mathbb{Q}_{v}$ are the completions of $K, \mathbb{Q}$ with respect to $v$. For archimedean $v$ we normalise the valuation by $|x|_{v}=|x|^{D_{v} / D}$ where $|\cdot|$ is the ordinary complex absolute value. When $v$ is non-archimedean we take $|p|_{v}=p^{-D_{v} / D}$ where $p$ is the unique rational prime such that $|p|_{v}<1$. The height of an algebraic number $\alpha \in K$ is defined by

$$
H(\alpha)=\prod_{v} \max \left(1,|x|_{v}\right) .
$$

Because of our normalisation $H(\alpha)$ does not depend on the choice of the field $K$ in which $\alpha$ is contained. We can now state Lehmer's conjecture.

Conjecture 1.1. There exists a number $c>1$ such that for any algebraic number $\alpha$, not a root of unity and of degree $D$ we have

$$
H(\alpha)^{D} \geq c .
$$

Presumably $c=1.1762808 \ldots$, which is the larger real root of $x^{10}+x^{9}-$ $x^{7}-x^{6}-x^{5}-x^{4}-x^{3}+x+1$.

The best unconditional result so far follows from work of Dobrowolski, Cantor \& Straus and Louboutin [Lo], stating that there exists $\gamma>0$ such that

$$
H(\alpha)^{D} \geq 1+\gamma\left(\frac{\log \log D}{\log D}\right)^{3}
$$

It came as a great surprise when S. Zhang [Zh] showed in 1992 that there does exist a number $c_{1}>1$ such that

$$
H(\alpha) H(1-\alpha) \geq c_{1}
$$


for all $\alpha \in \overline{\mathbb{Q}}$ such that $\alpha \neq 0,1, \frac{1}{2} \pm \frac{1}{2} \sqrt{-3}$. This was proved by using Arakelov intersection theory on $\mathbb{P}^{1}$. It was almost immediately realised by one of us (see $[\mathrm{Za}]$ ) that an elementary proof could be given which at the same time yields the best possible $c_{1}$, namely $\sqrt{\eta}$ where $\eta=(1+\sqrt{5}) / 2$, the golden ratio. The minimum is attained when $\alpha$ is minus a fifth root of unity. In $[\mathrm{Za}]$ there is also a generalisation of the following sort. For any $K$-rational point $P=\left(P_{0}: P_{1}: \ldots: P_{n}\right)$ in $n$-dimensional projective space $\mathbb{P}^{n}$ we define the height by

$$
H(P)=\prod_{v} \max \left(\left|P_{0}\right|_{v}, \ldots,\left|P_{n}\right|_{v}\right) .
$$

In particular, the height of an algebraic number $\alpha$ is nothing but the projective height of $(1: \alpha) \in \mathbb{P}^{1}(K)$. Then it is shown in [Za] that for any $\left(x_{0}: x_{1}: x_{2}\right) \in \mathbb{P}^{2}(\overline{\mathbb{Q}})$ such that $x_{0}+x_{1}+x_{2}=0, x_{0} x_{1} x_{2} \neq 0$ and $\left(x_{0}: x_{1}: x_{2}\right) \neq\left(1: \omega^{ \pm 1}: \omega^{\mp 1}\right)\left(\omega^{3}=1\right)$, we have

$$
H\left(x_{0}, x_{1}, x_{2}\right) \geq c_{2}
$$

where $c_{2}$ is the larger real root of $x^{6}-x^{4}-1$. The minimum is attained when the $x_{i}$ are the roots of $x^{3}+x-1$.

Inspired by $[\mathrm{Za}], \mathrm{H}$. P. Schlickewei and E. Wirsing [SW] showed the following result. Consider the line $L: \lambda x+\mu y+\nu z=0$ in $\mathbb{P}^{2}$ with $\lambda \mu \nu \neq 0$. Suppose that $\lambda+\mu+\nu=0$. Then, for any two points $P_{1}, P_{2} \in L(\overline{\mathbb{Q}})$ with non-zero coordinates and such that $(1: 1: 1), P_{1}, P_{2}$ are distinct, we have

$$
H\left(P_{1}\right) H\left(P_{2}\right)>\exp (1 / 2400)=1.00041 \ldots
$$

This result was applied by Schlickewei [Schl] to estimating numbers of solutions of three term $S$-unit equations in a strikingly successful way. Although very useful, the derivation of the Schlickewei-Wirsing result did not look optimal. It is the goal of this paper to remedy this situation and also give a generalisation which encompasses the previous results. We finish the introduction by giving a description of our general setup and main result.

Consider a hypersurface $S$ of multidegree $d_{1}, \ldots, d_{r}$ on $\mathbb{P}^{n_{1}} \times \ldots \times \mathbb{P}^{n_{r}}$ given by a polynomial $F$ with coefficients in $\mathbb{Z}$. Denote the coordinates of $\mathbb{P}^{n_{i}}$ by $\mathbf{x}_{i}=\left(x_{i 0}, x_{i 1}, \ldots, x_{i n_{i}}\right)$. The degree of $F$ in the variable $x_{i j}$ is denoted by $d_{i j}$. We define $\widetilde{d}_{i}=-d_{i}+\sum_{j} d_{i j}$.

Choose a subset $I$ of $\left\{i: n_{i}=1\right\}$ and let $E$ be the set $\{(i, 0): i \in I\}$, to which we refer as exceptional index pairs. For any polynomial with coefficients in $\mathbb{Z}$ we denote by $\|P\|$ the sum of the absolute values of the coefficients. We define

$$
c_{i j}=\left\|\frac{\partial F}{\partial x_{i j}}\right\|, \quad c_{F}=\max _{(i, j) \notin E} c_{i j} .
$$

The advantage of having the exceptional set $E$ is that the value of $c_{F}$ may 
be smaller than the one we would get by taking the maximum over all pairs $(i, j)$. In the first example in [Za] this enables us to get the optimal lower bound for the product $H(\alpha) H(1-\alpha)$. By $\delta$ we denote the maximum of the numbers $\max _{i \in I}\left(\widetilde{d}_{i}+d_{i, 1}\right) / 2$ and $\max _{i \notin I} \widetilde{d}_{i} /\left(n_{i}+1\right)$.

TheOREM 1.2. For each point $\left(\mathbf{x}_{1}, \ldots, \mathbf{x}_{r}\right) \in \mathbb{P}^{n_{1}}(\overline{\mathbb{Q}}) \times \ldots \times \mathbb{P}^{n_{r}}(\overline{\mathbb{Q}})$ such that $F\left(x_{i j}\right)=0, x_{i j} \neq 0$ for all $i, j$ and $F\left(x_{i j}^{-1}\right) \neq 0$ we have

$$
H\left(\mathbf{x}_{1}\right)^{n_{1}+1} \ldots H\left(\mathbf{x}_{r}\right)^{n_{r}+1} \geq \varrho,
$$

where $\varrho$ is the unique real root larger than 1 of $x^{-2}+c_{F}^{-1} x^{-\delta}=1$.

During the preparation of this paper W. M. Schmidt informed us that in $[\mathrm{Schm}]$ he had already proved a theorem very similar to ours in the case where one works in $\left(\mathbb{P}^{1}\right)^{r}$. The logarithm of the lower bound given in [Schm] is $1 /\left(2^{4 f+2 r} H\right)$, where $f$ is the total degree of $F$ and $H$ the maximum of all coefficients. Although the basic starting point in this paper and [Schm] is the same, we nevertheless found that the principle of our approach and the better values of the constants have some interest.

2. Applications. Before proving the theorem we describe a few consequences. First of all consider $r$ algebraic numbers $\alpha_{1}, \ldots, \alpha_{r}$ whose sum is a rational integer $N$. We like to interpret the $r$-tuple as a point $(1$ : $\left.\alpha_{1}\right) \times \ldots \times\left(1: \alpha_{r}\right) \in\left(\mathbb{P}^{1}\right)^{r}$. For the set $I$ of our theorem we choose $\{1, \ldots, r\}$. Letting $F$ be the homogeneous version of $x_{1}+\ldots+x_{r}-N$ one easily checks that $c_{i}=1$ for all $i$. Note that the coefficient $N$ in $F$ does not appear in the $c_{i}$ because of our choice of $I$. So we get $c_{F}=1$. Moreover, $n_{i}=1$ and $d_{i}=1$ for all $i$. Hence $\delta=1$. Thus we find

Corollary 2.1. Let $\alpha_{1}, \ldots, \alpha_{r} \in \overline{\mathbb{Q}}^{*}$ and $N \in \mathbb{Z}$ be such that $\alpha_{1}+\ldots+$ $\alpha_{r}=N$ and $\alpha_{1}^{-1}+\ldots+\alpha_{r}^{-1} \neq N$. Then

$$
H\left(\alpha_{1}\right) \ldots H\left(\alpha_{r}\right) \geq \sqrt{\eta}
$$

where $\eta$ is the golden ratio.

Note that when $r \geq 4$ the lower bound is actually attained for the $r$-tuple $-\zeta_{5}, 1+\zeta_{5}, 1, \zeta_{r-2}, \ldots, \zeta_{r-2}^{r-3}$ where $\zeta_{k}$ denotes a primitive $k$ th root of unity. When we take for the $\alpha_{i}$ the conjugates of an algebraic number $\alpha$ of degree $D$ we get the following consequence.

Corollary 2.2. Let $\alpha \in \overline{\mathbb{Q}}^{*}$ be such that trace $(\alpha)$ is integral and trace $(\alpha)$ $\neq \operatorname{trace}\left(\alpha^{-1}\right)$. Then $H(\alpha)^{D} \geq \sqrt{\eta}$.

However, this result is already contained in a result of C. Smyth [Sm] which states that $H(\alpha)^{D} \geq \theta$ for every non-reciprocal $\alpha \in \overline{\mathbb{Q}}^{*}$. Here $\theta$ is the real root of $x^{3}-x-1$. 
We now consider $r$ algebraic numbers $\alpha_{i}$ whose sum is 1 and give a lower bound for $H\left(1, \alpha_{1}, \ldots, \alpha_{r}\right)$. The polynomial $F$ can be written as $x_{1}+\ldots+$ $x_{r}-x_{0}$ and we have $c_{F}=1, d_{1}=1$. Furthermore, $\delta=r /(r+1)$.

Corollary 2.3. For any $\alpha_{1}, \ldots, \alpha_{r} \in \overline{\mathbb{Q}}^{*}$ such that $\alpha_{1}+\ldots+\alpha_{r}=1$ and $\alpha_{1}^{-1}+\ldots+\alpha_{r}^{-1} \neq 1$ we have

$$
H\left(1, \alpha_{1}, \ldots, \alpha_{r}\right) \geq \varrho
$$

where $\varrho$ is the real root larger than 1 of $1=x^{-2 r-2}+x^{-r}$.

As pointed out in the introduction, this result is optimal when $r=2$. For $r>2$ this is not true any more. When $r=3$ for example we find the lower bound $1.14613 \ldots$ (which improves the bound $\exp (1 / 402)=1.00249 \ldots$ from $[\mathrm{SW}])$. However, the lowest height we could find was $H=1.15096 \ldots$ when the $\alpha_{i}$ are the zeros of $x^{3}-x^{2}+1$. On the other hand, the asymptotic behaviour of $\varrho$ as a function of $r$ looks optimal. It is not hard to show that $\varrho^{r+1} \rightarrow \eta$ as $r \rightarrow \infty$ while on the other hand the zeros $\alpha_{0}, \ldots, \alpha_{r}$ of $x^{r+1}-x-1$ satisfy $H\left(\alpha_{0}, \ldots, \alpha_{r}\right)^{r+1} \rightarrow 2$ as $r \rightarrow \infty$.

We now consider the Schlickewei-Wirsing result. Suppose we have a line $L: \lambda x+\mu y+\nu z=0$ in $\mathbb{P}^{2}$ with $\lambda \mu \nu \neq 0$. Let $P_{1}, P_{2}, P_{3} \in L(\overline{\mathbb{Q}})$ be three distinct points with non-zero coordinates. Letting $P_{i}=\left(x_{i}: y_{i}: z_{i}\right)$ $(i=1,2,3)$ we get the relation

$$
\Delta:=\left|\begin{array}{lll}
x_{1} & y_{1} & z_{1} \\
x_{2} & y_{2} & z_{2} \\
x_{3} & y_{3} & z_{3}
\end{array}\right|=0 .
$$

We want a lower bound of $H\left(P_{1}\right) H\left(P_{2}\right) H\left(P_{3}\right)$. Our polynomial $F$ is now the determinant form $\Delta$. First we point out that

$$
\widetilde{\Delta}:=\left|\begin{array}{lll}
x_{1}^{-1} & y_{1}^{-1} & z_{1}^{-1} \\
x_{2}^{-1} & y_{2}^{-1} & z_{2}^{-1} \\
x_{3}^{-1} & y_{3}^{-1} & z_{3}^{-1}
\end{array}\right| \neq 0 .
$$

Suppose $\widetilde{\Delta}=0$. Then there exist $\alpha, \beta, \gamma$, not all zero, such that $\alpha x_{i}^{-1}+$ $\beta y_{i}^{-1}+\gamma z_{i}^{-1}=0$ for $i=1,2,3$. Hence $\alpha y_{i} z_{i}+\beta z_{i} x_{i}+\gamma x_{i} y_{i}=0(i=1,2,3)$. The conic $C: \alpha y z+\beta z x+\gamma x y=0$ is reducible if and only if $\alpha \beta \gamma=0$. So, if $\gamma=0$ for example, we get $\alpha x_{i}+\beta y_{i}=0$ for $i=1,2,3$. But this contradicts $\nu \neq 0$. So $C$ is an irreducible conic. But then $P_{1}, P_{2}, P_{3}$ lie both on $C$ and $L$, which is impossible since $|C \cap L| \leq 2$. We conclude that $\widetilde{\Delta} \neq 0$. We can now apply our theorem with $r=3, n_{1}=n_{2}=n_{3}=2, d_{1}=d_{2}=d_{3}=1, c_{F}=2$ and $I=\emptyset$.

Corollary 2.4. Consider the line $L: \lambda x+\mu y+\nu z=0$ in $\mathbb{P}^{2}$ with $\lambda \mu \nu \neq$ 0. Let $P_{1}, P_{2}, P_{3} \in L(\overline{\mathbb{Q}})$ be three distinct points with non-zero coordinates. 
Then

$$
H\left(P_{1}\right) H\left(P_{2}\right) H\left(P_{3}\right) \geq \varrho,
$$

where $\varrho$ is the real root larger than 1 of $1=\varrho^{-6}+(1 / 2) \varrho^{-2}$.

The numerical value of $\varrho$ is $1.09427 \ldots$, which compares favourably with the value $1.00041 \ldots$ from $[\mathrm{SW}]$ or $1.019 \ldots$ from $[\mathrm{Schm}]$. Moreover, this result was applied successfully to equations of the form $x+y=1$ with $x, y$ unknowns in a finitely generated multiplicative group and to multiplicity estimates for binary recurrences in [BS].

3. Proof of Theorem 1.2. The proof is based on the following observation, which is a direct generalisation of [Za]. Let $X$ be a closed subvariety of $\mathbb{P}^{n_{1}} \times \ldots \times \mathbb{P}^{n_{r}}$ defined over $\mathbb{Q}$. We denote the coordinates by $\mathbf{x}=\left(\mathbf{x}_{1}, \ldots, \mathbf{x}_{r}\right)$ with $\mathbf{x}_{i}=\left(x_{i 0}, \ldots, x_{i n_{i}}\right)$. Denote by $X(\mathbb{C})_{1}$ the intersection of $X(\mathbb{C})$ with the polydisc $\left\{\mathbf{x}:\left|x_{i j}\right| \leq 1 \forall i, j\right\}$. We also give ourselves a collection of multihomogeneous polynomials $G_{k}(\mathbf{x}) \in \mathbb{Z}[\mathbf{x}]$ of multidegrees $\left(d_{k 1}, \ldots, d_{k r}\right)$.

LEMMA 3.1. Let $\nu_{k} \geq 0$ for all $k$ and set

$$
w_{i}=\sum_{k} \nu_{k} d_{k i}, \quad \lambda=-\max _{\mathbf{x} \in X(\mathbb{C})_{1}}\left\{\sum_{k} \nu_{k} \log \left|G_{k}(\mathbf{x})\right|\right\} .
$$

Then for any point $\mathbf{x}=\left(\mathbf{x}_{1}, \ldots, \mathbf{x}_{r}\right) \in X(\overline{\mathbb{Q}})$ with $\prod_{k} G_{k}(\mathbf{x}) \neq 0$ we have

$$
\prod_{i=1}^{r} H\left(\mathbf{x}_{i}\right)^{w_{i}} \geq e^{\lambda}
$$

Proof. Suppose that $\mathbf{x} \in X(K)$ with $G_{k}(\mathbf{x}) \neq 0$ for all $k$. Here $K$ is an algebraic number field of degree $D$, say. For any valuation $v$ of $K$ we let $D_{v}=\left[K_{v}: \mathbb{Q}_{v}\right]$. Then the inequality

$$
\sum_{i=1}^{r} w_{i} \log \left(\max _{j}\left|x_{i j}\right|_{v}\right) \geq \sum_{k} \nu_{k} \log \left|G_{k}(\mathbf{x})\right|_{v}+ \begin{cases}\frac{D_{v}}{D} \lambda & \text { if } v \mid \infty, \\ 0 & \text { if } v \nmid \infty,\end{cases}
$$

holds for all places $v$ of $K$, because by the homogeneity condition (1) we may assume that $\max _{j}\left|x_{i j}\right|_{v}=1$ for all $i$ and the inequality follows from the definition of $\lambda$ if $v$ is infinite and is straightforward if $v$ is finite. The lemma follows by summing over all $v$ and using the product formula.

The following lemma saves us a considerable amount of effort in the determination of $\lambda$ for the sake of the previous lemma.

LEMMA 3.2. With notations as above, the function $\Psi:=\sum \nu_{k} \log \left|G_{k}(\mathbf{x})\right|$ assumes a maximum in $\mathbf{x} \in X(\mathbb{C})_{1}$ and it is attained at a point all of whose coordinates have absolute value 1 with at most one exception.

Proof. Since the $\nu_{k}$ are positive, $\Psi$ is bounded from above in $X(\mathbb{C})_{1}$. For $\varepsilon>0$ sufficiently small the set $\mathbf{x} \in X(\mathbb{C})_{1}$ such that $\Psi(\mathbf{x}) \geq \log (\varepsilon)$ is 
compact and not empty. Hence it is clear that $\Psi$, being continuous, assumes a maximum.

Now suppose that $\Psi$ assumes a maximum at a point $P$ where at least two coordinates have absolute value $<1$. Call these coordinates $\xi, \eta$ and denote the values of these coordinates at $P$ by $\xi_{0}, \eta_{0}$. Substitute in $F=0$ the values of all coordinates of $P$ except $\xi, \eta$. The equation $F=0$ reduces to the equation of a curve $f(\xi, \eta)=0$ containing the point $\xi_{0}, \eta_{0}$. By choosing a branch of $f=0$ at the point $\xi_{0}, \eta_{0}$ we find locally analytic functions $\xi(t), \eta(t)$ such that $\xi(0)=\xi_{0}, \eta(0)=\eta_{0}$ and $f(\xi(t), \eta(t))=0$ identically in a neighbourhood of $t=0$. When $f$ was identically zero anyway, we can choose $\xi(t), \eta(t)$ arbitrarily. Choose a disk $D$ in the complex $t$-plane around 0 such that $|\xi(t)|,|\eta(t)| \leq 1$ for all $t \in D$. Specialise the arguments in $\Psi$ to the values of the point $P$ except for $\xi$ and $\eta$ where we substitute $\xi(t)$ and $\eta(t)$. In this way we obtain a function $\psi(t)$ in $t \in D$ which assumes a maximum at $t=0$. Notice that $\psi(t)$ is harmonic in the real and imaginary part of $t$. A harmonic function assuming a maximum in the interior of its domain is necessarily constant. Hence $\psi(t)$ is constant. But in that case we can continue $\xi(t)$ and $\eta(t)$ analytically until either one of them hits the unit circle. At that new point the value of $\Psi$ is again $\psi(0)$, i.e. maximal. We continue this procedure for other coordinates, if necessary, until we have found an optimal point all of whose coordinates have absolute value one with at most one exception.

LEMma 3.3. Let $\alpha, \beta, \gamma>0$. Let $m$ be the unique minimum of the function

$$
u \log \frac{\gamma u}{u+v}+v \log \frac{v}{u+v}
$$

under the constraints $u, v \geq 0, \alpha u+\beta v=1$. Then $e^{-m}$ is the unique real root larger than 1 of $\gamma^{-1} x^{-\alpha}+x^{-\beta}=1$.

Proof. Put $x=v /(u+v)$ and $1-x=u /(u+v)$. Then

$$
u=\frac{1-x}{\beta x+\alpha(1-x)}, \quad v=\frac{x}{\beta x+\alpha(1-x)}
$$

and $x \in[0,1]$. We must minimize

$$
f(x)=\frac{(1-x) \log (\gamma(1-x))+x \log x}{\beta x+\alpha(1-x)}
$$

on $[0,1]$. Differentiate with respect to $x$,

$$
f^{\prime}(x)=\frac{-\beta \log (\gamma(1-x))+\alpha \log x}{(\beta x+\alpha(1-x))^{2}} .
$$

This vanishes if $(\gamma(1-x))^{\beta}=x^{\alpha}$. Since $x$ is strictly increasing and $1-x$ strictly decreasing there is a unique solution $\left.x_{0} \in\right] 0,1[$. Choose $\varrho>0$ such that $x_{0}=\varrho^{-\beta}$. Then $\gamma(1-x)=\varrho^{-\alpha}$ and thus we see that $\varrho$ satisfies 
$1-\varrho^{-\beta}=\gamma^{-1} \varrho^{-\alpha}$. It remains to verify that $f\left(x_{0}\right)=-\log \varrho$, which is straightforward.

Pro of of Theorem 1.2. We apply Lemma 3.1 to the hypersurface $X$ given by the multihomogeneous polynomial $F(\mathbf{x}) \in \mathbb{Z}[\mathbf{x}]$ with multidegrees $d_{1}, \ldots, d_{r}$. For the $G_{k}$ we take the coordinates $x_{i j}$ and the function

$$
\widetilde{F}(\mathbf{x})=F\left(x_{i j}^{-1}\right) \prod x_{i j}^{d_{i j}}
$$

where $d_{i j}$ is the degree of $F$ in $x_{i j}$. Let $\mu, \nu_{i j} \geq 0$. Let $\Phi(\mathbf{x})$ be the function $\mu \log |\widetilde{F}(\mathbf{x})|+\sum_{i, j} \nu_{i j} \log \left|x_{i j}\right|$ on $X(\mathbb{C})$. Let $\widetilde{d}_{i}=-d_{i}+\sum_{j} d_{i j}$ be the degree of $\widetilde{F}$ in $\mathbf{x}_{i}$ and suppose

$$
w_{i}=\mu \widetilde{d}_{i}+\sum_{j} \nu_{i j}, \quad \lambda=-\max _{\mathbf{x} \in X(\mathbb{C})_{1}} \Phi(\mathbf{x}) .
$$

Then Lemma 3.1 states that $(2)$ holds for all $\mathbf{x} \in X(\overline{\mathbb{Q}})$ with $x_{i j} \neq 0$ and $F\left(x_{i j}^{-1}\right) \neq 0$.

Let us take $w_{i}=n_{i}+1$ for all $i$. Although there are many other choices for the weights $w_{i}$, this choice gives us the particularly simple shape of our main theorem. It remains to choose $\mu, \nu_{i j}$ in such a way that $\lambda$ becomes positive and as large as possible. We choose

$$
\nu_{i j}=1-\frac{\widetilde{d}_{i}}{n_{i}+1} \mu \quad \text { if } i \notin I
$$

and

$$
\nu_{i, 0}=1-\frac{\widetilde{d}_{i}-d_{i, 1}}{2} \mu, \quad \nu_{i, 1}=1-\frac{\widetilde{d}_{i}+d_{i, 1}}{2} \mu \quad \text { if } i \in I .
$$

Let us determine $\max _{\mathbf{x} \in X(\mathbb{C})_{1}} \Phi(\mathbf{x})$. By Lemma 3.2 this maximum is attained at a point all of whose coordinates, with possibly one exception, lie on the unit circle. Suppose that $\left|x_{i_{0} j_{0}}\right| \leq 1$ and that $\left|x_{i j}\right|=1$ for all $(i, j) \neq\left(i_{0}, j_{0}\right)$. Suppose first that $\left(i_{0}, j_{0}\right) \notin E$. Then

$$
\begin{aligned}
\left|\widetilde{F}\left(x_{i j}\right)\right| & =\left|F\left(x_{i j}^{-1}\right)\right| \cdot\left|\prod_{i, j}\left(x_{i j}\right)^{d_{i j}}\right|=\left|F\left(x_{i_{0} j_{0}}^{-1}, \bar{x}_{i j}\right)\right| \cdot\left|x_{i_{0} j_{0}}\right|^{d_{i_{0} j_{0}}} \\
& =\left|F\left(x_{i_{0} j_{0}}^{-1}, \bar{x}_{i j}\right)-F\left(\bar{x}_{i j}\right)\right| \cdot\left|x_{i_{0} j_{0}}\right|^{d_{i_{0} j_{0}}} \\
& \leq c_{i_{0} j_{0}}\left|x_{i_{0} j_{0}}^{-1}-\bar{x}_{i_{0} j_{0}}\right| \max \left(\left|x_{i_{0} j_{0}}\right|^{-1},\left|x_{i_{0} j_{0}}\right|\right)^{d_{i_{0} j_{0}}-1} \cdot\left|x_{i_{0} j_{0}}\right|^{d_{i_{0} j_{0}}} \\
& =c_{i_{0} j_{0}}\left(1-\left|x_{i_{0} j_{0}}\right|^{2}\right) .
\end{aligned}
$$

Put $\left|x_{i_{0} j_{0}}\right|^{2}=\xi$. We see that the maximum of $\Phi$ is

$$
\max _{\xi \in[0,1]}\left[\mu \log \left(c_{i_{0} j_{0}}(1-\xi)\right)+\left(\nu_{i_{0} j_{0}} / 2\right) \log \xi\right] .
$$


This maximum is attained at $\xi=\nu_{i_{0} j_{0}} /\left(\nu_{i_{0} j_{0}}+2 \mu\right)$ and its value is

$$
\mu \log \frac{2 \mu c_{i_{0} j_{0}}}{\nu_{i_{0} j_{0}}+2 \mu}+\frac{\nu_{i_{0} j_{0}}}{2} \log \frac{\nu_{i_{0} j_{0}}}{\nu_{i_{0} j_{0}}+2 \mu} .
$$

Since we have $\nu_{i_{0} j_{0}} \geq 1-\delta \mu$, this maximum is bounded above by

$$
\mu \log c_{F} \frac{2 \mu}{(1-\delta \mu)+2 \mu}+\frac{1-\delta \mu}{2} \log \frac{1-\delta \mu}{(1-\delta \mu)+2 \mu} .
$$

We now determine the maximum when $\left(i_{0}, j_{0}\right) \in E$. In particular, $j_{0}=0$. So suppose we have $\left|x_{i_{0}, 0}\right| \leq 1$ and $\left|x_{i j}\right|=1$ for all other $i, j$. Writing down the dependence on $x_{i_{0}, 0}, x_{i_{0}, 1}$ explicitly and putting $z=x_{i_{0}, 0} / x_{i_{0}, 1}$, we find

$$
\begin{aligned}
\left|\widetilde{F}\left(x_{i j}\right)\right| & =\left|F\left(x_{i_{0}, 0}^{-1}, x_{i_{0}, 1}^{-1}, x_{i j}^{-1}\right)\right| \cdot\left|x_{i_{0}, 0}\right|^{d_{i_{0}, 0}}=\left|F\left(1, z, x_{i j}^{-1}\right)\right| \\
& =\left|F\left(1, z, \bar{x}_{i j}\right)-F\left(1,1 / \bar{z}, \bar{x}_{i j}\right)\right| \\
& \leq c_{i_{0}, 1}|z-1 / \bar{z}| \max \left(|z|,|z|^{-1}\right)^{d_{i_{0}, 1}-1} \\
& =\left.\left.c_{i_{0}, 1}|1-| z\right|^{2}|\cdot| z\right|^{-d_{i_{0}, 1}} .
\end{aligned}
$$

Put $\xi=|z|^{2}=\left|x_{i_{0}, 0}\right|^{2}$. We see that the maximum of $\Phi$ is

$$
\max _{\xi \in[0,1]}\left[\mu \log \left(c_{i_{0}, 1}(1-\xi)\right)-\left(d_{i_{0}, 1} \mu / 2\right) \log |\xi|+\left(\nu_{i_{0}, 0} / 2\right) \log |\xi|\right],
$$

which equals

$$
\mu \log \frac{2 c_{i_{0} j_{0}} \mu}{\widetilde{\nu}+2 \mu}+\frac{\widetilde{\nu}}{2} \log \frac{\widetilde{\nu}}{\widetilde{\nu}+2 \mu}
$$

where $\widetilde{\nu}=\nu_{i_{0}, 0}-d_{i_{0}, 1} \mu / 2$. Note that by our choice of $\nu_{i_{0}, 0}$,

$$
\widetilde{\nu}=1-\left(\widetilde{d}_{i_{0}}+d_{i_{0}, 1}\right) \mu / 2 \geq 1-\delta \mu .
$$

Hence our maximum is again bounded by $(\mathrm{M})$. Now use Lemma 3.3 with $\alpha=\delta, \beta=2, \gamma=c_{F}$ to minimize (M) by letting $\mu$ vary. The assertion of our theorem follows immediately.

\section{References}

[BS] F. Beukers and H. P. Schlickewei, The equation $x+y=1$ in finitely generated groups, Acta Arith. 78 (1996), 189-199.

[Le] D. H. Lehmer, Factorisation of certain cyclotomic functions, Ann. of Math. 34 (1933), 461-479.

[Lo] R. Louboutin, Sur la mesure de Mahler d'un nombre algébrique, C. R. Acad. Sci. Paris 296 (1983), 707-708.

[Schl] H. P. Schlickewei, Equations $a x+b y=1$, Ann. of Math., to appear.

[SW] H. P. Schlickewei and E. Wirsing, Lower bounds for the heights of solutions of linear equations, Invent. Math., to appear.

[Schm] W. M. Schmidt, Heights of algebraic points lying on curves or hypersurfaces, Proc. Amer. Math. Soc., to appear. 
[Sm] C. J. Smyth, On the product of the conjugates outside the unit circle of an algebraic integer, Bull. London Math. Soc. 3 (1971), 169-175.

[Za] D. Zagier, Algebraic numbers close to both 0 and 1, Math. Comp. 61 (1993), 485-491.

[Zh] S. Zhang, Positive line bundles on arithmetic surfaces, Ann. of Math. 136 (1992), 569-587.

Department of Mathematics

University of Utrecht

Budapestlaan 6

P.O. Box 80010

3508 TA Utrecht, The Netherlands

E-mail: beukers@math.ruu.nl
Max-Planck-Institut für Mathematik

Gottfried-Claren-Str. 26 53225 Bonn, Germany 